\title{
Completeness of colon and rectal cancer staging in the Danish Cancer Registry, 2004-2009
}

This article was published in the following Dove Press journal:

Clinical Epidemiology

16 August 2012

Number of times this article has been viewed

\author{
Eva Bjerre Ostenfeld ${ }^{1,2}$ \\ Trine Frøslev' \\ Søren Friis ${ }^{3}$ \\ Per Gandrup ${ }^{2}$ \\ Mogens Rørbæk Madsen ${ }^{4}$ \\ Mette Søgaard ${ }^{1,5}$ \\ 'Department of Clinical Epidemiology, \\ Aarhus University Hospital, Aarhus, \\ Denmark; ${ }^{2}$ Department of Surgery A, \\ Aarhus University Hospital, Aalborg, \\ Denmark; ${ }^{3}$ Danish Cancer Society \\ Research Center, Danish Cancer \\ Society, Copenhagen, Denmark; \\ ${ }^{4}$ Department of Surgery A, Herning \\ Regional Hospital, Herning, Denmark; \\ ${ }^{5}$ Department of Clinical Microbiology, \\ Aarhus University Hospital, Aalborg, \\ Denmark
}

Objective: To explore the completeness of tumor, node, metastasis (TNM) staging for colon and rectal cancer in the Danish Cancer Registry.

Material and methods: From the Danish Cancer Registry, we retrieved data on TNM stage, year of diagnosis, sex, and age for 15,976 and 8292 patients, respectively, with first diagnoses of colon or rectal cancer during the 2004-2009 period. From the Danish National Patient Register, we retrieved data on comorbidity (computed as Charlson Comorbidity Index scores). We calculated the completeness of TNM staging overall, by each stage component, and according to a stage algorithm allowing some missing stage components. Analyses were stratified by sex, age, year of diagnosis, and Charlson Comorbidity Index score.

Results: For colon and rectal cancer, overall TNM completeness was 67.8\% (95\% confidence interval [CI]: 67.0\%-68.5\%) and 68.1\% (95\% CI: 67.0\%-69.1\%), respectively. For both cancers, completeness decreased with increasing age and level of comorbidity, whereas differences between the sexes were minor. Over the study period, TNM completeness for colon cancer decreased from $71.3 \%$ (95\% CI: 69.5\%-73.0\%) to 64.8\% (95\% CI: 63.0\%-66.6\%), whereas the completeness for rectal cancer remained stable over time. When using the stage algorithm, the completeness rose markedly, to $81.1 \%$ for colon cancer and $79.0 \%$ for rectal cancer.

Conclusion: One-third of colon and rectal cancer cases in the Danish Cancer Registry had missing TNM stage information, which varied with age and level of comorbidity. Cancer cases with unknown staging warrant serious consideration of the methodological implications in future epidemiological studies monitoring cancer incidence and outcomes.

Keywords: colorectal neoplasm, neoplasm staging, TNM, registries, epidemiology, cancer

\section{Introduction}

Colorectal cancer (CRC) is the third most common cancer worldwide and the fourth most common cause of cancer-related death. ${ }^{1}$ Tumor stage is a key determinant of CRC prognosis and provides guidance to the optimal planning of treatment. Furthermore, the stage is important for monitoring trends in CRC incidence and mortality across populations. The tumor, node, metastasis (TNM) stage classification is based on the anatomic extent of the tumor, including the tumor size $(\mathrm{T})$, the number of lymph nodes involved $(\mathrm{N})$, and the presence of metastases $(\mathrm{M}){ }^{2}$

Since 1943, all incident cancers in Denmark have been recorded in the Danish Cancer Registry (DCR). ${ }^{3,4}$ Reporting to the DCR has been mandatory since 1987, and ascertainment of cancer cases in the registry is virtually complete. ${ }^{3-5}$ TNM staging has been recorded for cancer cases since 2004. ${ }^{4}$ However, no studies have hitherto examined the completeness of TNM staging in the DCR. Some studies have suggested that factors such as age, race, sex, marital status, income, and residence influence the
Correspondence: Eva Bjerre Ostenfeld, Department of Clinical Epidemiology, Aarhus University Hospital, Olof Palmes Allé 43-45, 8200 Aarhus N, Denmark Tel +4587168238

Fax +458716 7215

Emaileos@dce.au.dk 
proportion of unstaged cancers. ${ }^{6-8}$ Given that information on TNM might not be missing at random, unstaged CRCs could bias results of studies monitoring cancer incidence and outcomes. We therefore aimed to evaluate the completeness of CRC staging in the DCR according to the TNM classification - overall, and by sex, age, year of diagnosis, and level of comorbidity.

\section{Methods}

We performed this study in Denmark, within a population of 5.4 million inhabitants. The Danish National Health Service provides free medical care by general practitioners and hospitals. All health-related services are registered with a unique ten-digit personal identifier - the CPR number - assigned since 1968 to each resident. ${ }^{9}$ This number allows unambiguous individual-level data linkage between Danish registers.

\section{Ascertaining patients with CRC}

We used the DCR to identify patients with a primary diagnosis of CRC between January 1, 2004 and December 31, 2009. During this period, the DCR recorded cancer diagnoses according to the International Classification of Disease, 10th revision (ICD-10). ${ }^{3,4}$ Colon and rectal cancer cases were identified by the ICD-10 codes C18 and C19-20, respectively. From the DCR, we also obtained information on CPR number, date of diagnosis, age, sex, and TNM stage at diagnosis.

\section{Comorbidity data}

The Danish National Patient Register contains data on all nonpsychiatric discharges from hospitals in Denmark since 1977 and all outpatient visits since $1995 .{ }^{10}$ Information includes CPR number, date of contact/discharge, and diagnoses according to ICD-10 since 1994. From the Danish National Patient Register, we obtained information on preexisting comorbidity 10 years prior to the date of CRC diagnosis using a modified version of the Charlson Comorbidity Index (CCI). The CCI is based on disease categories that are each weighted according to the adjusted risk of one-year mortality. ${ }^{11,12}$ Excluding CRC from the index, we defined the level of comorbidity as low (CCI score $=0)$, medium (CCI score $=1-2)$, and high $($ CCI score $\geq 3)$.

\section{Statistical analysis}

We calculated the completeness of TNM stage registration and the corresponding 95\% confidence intervals (CIs), both overall and for each component individually (ie, T, N, and M). The completeness was defined as the number of individuals with no missing factors (ie, $\mathrm{T} 1-4, \mathrm{~N} 0-3$, and $\mathrm{M} 0-1$ ) divided by the total number of patients. We stratified completeness by sex, age (0-39 years, 40-59 years, 60-79 years, and $\geq 80$ years), year of colon or rectal cancer diagnosis, and CCI score.

Complete information on $\mathrm{T}, \mathrm{N}$, and $\mathrm{M}$ is necessary to derive a definite TNM stage in the DCR. For additional categorization of colon or rectal cancers into localized, regional, distant, or unknown stages, we designed an algorithm, allowing certain missing stage components, under the assumption that the remaining information was sufficient to provide a meaningful categorization (eg, cancers assigned T4, Nx, M1 in the DCR were categorized as "distant"; see Appendix 1). The algorithm was based on knowledge of tumor growth and clinical coding practice In addition, we restricted the analysis to histologic verified CRC cases.

Analyses were performed using SAS (v 9.2; SAS Institute, Inc, Cary, NC).

\section{Results \\ Colon cancer}

A total of 15,976 patients were diagnosed with colon cancer during the 2004-2009 period (Table 1). Females accounted for $51.5 \%$ of the colon cancer cases, with a median age at diagnosis of 74 years. The median age for men was 72 years. Overall TNM completeness was $67.8 \%$ (95\% CI: 67.0\%-68.5\%) (Table 1). Examining each stage component, the overall registration proportion was slightly higher for M $(83.5 \%$ [95\% CI: $82.9 \%-84.0 \%$ ]) than for T $(80.3 \%$ [95\% CI: $79.7 \%-80.9 \%$ ]) and $\mathrm{N}(76.4 \%$ [95\% CI: 75.8\%-77.1\%]) (Table 2). We found that $93.3 \%$ of the colon cancer cases were histologically verified. Restricting to this proportion, overall TNM was $70.8 \%$ (95\% CI: 70.1\%-71.5\%).Differences in TNM completeness between the sexes were minor, with males exhibiting a slightly higher completeness than females. Completeness of the TNM staging decreased with (1) increasing age, from $68.5 \%(95 \%$ CI: $61.2 \%-75.1 \%$ ) in patients $<40$ years to $57.0 \%$ (95\% CI: $55.5 \%-58.5 \%$ ) in patients $\geq 80$ years; (2) year of diagnosis, from $71.3 \%$ (95\% CI: $69.5 \%-73.0 \%$ ) in 2004 to $64.8 \%$ (95\% CI: $63.0 \%-66.6 \%$ ) in 2009; and (3) level of comorbidity, from $70.8 \%$ (95\% CI: $69.9 \%-71.7 \%$ ) in patients with lowest comorbidity (CCI score $=0)$ to $57.2 \%(95 \% \mathrm{CI}: 54.8 \%-59.5 \%)$ among those with high level of comorbidity (CCI score $\geq 3$ ) (Table 1). Using the algorithm for stage classification (Appendix 1), we found that 5473 (34.3\%) of the colon cancers were localized, whereas regional and distant cases accounted for $3463(21.7 \%)$ and $4022(25.2 \%)$, respectively. A total of 3018 (18.9\%) colon cancers were not classifiable according to the algorithm (data not shown). 
Table I TNM completeness for colon and rectal cancer; overall and by sex, age, year, and comorbidity

\begin{tabular}{|c|c|c|c|c|c|c|c|c|}
\hline & \multicolumn{4}{|c|}{ Colon Cancer } & \multicolumn{4}{|c|}{ Rectal cancer } \\
\hline & \multirow{2}{*}{$\frac{\text { Total }}{\text { No. }}$} & \multicolumn{3}{|c|}{ TNM completeness } & \multirow{2}{*}{$\frac{\text { Total }}{\text { No. }}$} & \multicolumn{3}{|c|}{ TNM completeness } \\
\hline & & No. & $\%$ & $(95 \% \mathrm{Cl})$ & & No. & $\%$ & $(95 \% \mathrm{Cl})$ \\
\hline Overall & 15,976 & 10,824 & 67.8 & $(67.0-68.5)$ & 8292 & 5643 & 68.1 & $(67.0-69.1)$ \\
\hline \multicolumn{9}{|l|}{ Sex } \\
\hline Female & 8233 & 5534 & 67.2 & $(66.2-68.2)$ & 3341 & 2180 & 65.3 & $(63.6-66.9)$ \\
\hline Male & 7743 & 5290 & 68.3 & $(67.3-69.4)$ & 4951 & 3463 & 70.0 & $(68.7-7 I .2)$ \\
\hline \multicolumn{9}{|l|}{ Age } \\
\hline$\leq 39$ years & 168 & 115 & 68.5 & $(61.2-75.1)$ & 63 & 65 & 78.3 & $(68.6-86.1)$ \\
\hline $40-59$ years & 2327 & 1737 & 74.6 & $(72.9-76.4)$ & 1667 & 1266 & 75.9 & $(73.9-78.0)$ \\
\hline $60-79$ years & 9262 & 6568 & 70.9 & $(70.0-71.8)$ & 4926 & 3517 & 71.4 & (70.1-72.7) \\
\hline$\geq 80$ years & 4219 & 2404 & 57.0 & $(55.5-58.5)$ & 1616 & 795 & 49.2 & $(46.8-51.6)$ \\
\hline \multicolumn{9}{|c|}{ Year of diagnosis } \\
\hline 2004 & 2555 & 1822 & 71.3 & $(69.5-73.0)$ & 1339 & 930 & 69.5 & $(67.0-71.9)$ \\
\hline 2005 & 2615 & 1782 & 68.1 & $(66.3-69.9)$ & 1284 & 858 & 66.8 & $(64.2-69.4)$ \\
\hline 2006 & 2704 & 1885 & 69.7 & $(68.0-7 \mid .4)$ & 1417 & 962 & 67.9 & $(65.4-70.3)$ \\
\hline 2007 & 2656 & 1802 & 67.9 & $(66.1-69.6)$ & $137 \mid$ & 950 & 69.3 & (66.8-7I.7) \\
\hline 2008 & 2706 & 1757 & 64.9 & $(63.1-66.7)$ & 1442 & 938 & 65.1 & $(62.6-67.5)$ \\
\hline 2009 & 2740 & 1776 & 64.8 & $(63.0-66.6)$ & 1439 & 1005 & 69.8 & $(67.4-72.2)$ \\
\hline \multicolumn{9}{|c|}{ Comorbidity $^{a}$} \\
\hline Low & 9617 & 6806 & 70.8 & (69.9-7I.7) & 5464 & 3903 & 71.4 & $(70.2-72.6)$ \\
\hline Medium & 4706 & 3073 & 65.3 & $(63.9-66.7)$ & 2192 & 1409 & 64.3 & $(62.3-66.3)$ \\
\hline High & 1653 & 945 & 57.2 & (54.8-59.5) & 636 & 331 & 52.0 & $(48.2-55.9)$ \\
\hline
\end{tabular}

Notes: aLevel of comorbidity according to the Charlson comorbidity index $(\mathrm{CCl})$ score; Low $(\mathrm{CCl}$ score $=0)$, Medium $(\mathrm{CCl}$ score $=\mathrm{I}-2)$, High $(\mathrm{CCl}$ score $\geq 3)$.

Abbreviations: $\mathrm{Cl}$, confidence interval; TNM, tumor, node, metastasis.

Table $2 \mathrm{~T}, \mathrm{~N}$, and $\mathrm{M}$ completeness for colon cancer; overall and by sex, age, year, and comorbidity

\begin{tabular}{|c|c|c|c|c|c|c|c|c|c|c|}
\hline & \multirow{2}{*}{$\frac{\text { Total }}{\text { No. }}$} & \multicolumn{3}{|c|}{$\mathrm{T}$ completeness } & \multicolumn{3}{|c|}{$\mathbf{N}$ completeness } & \multicolumn{3}{|c|}{ M completeness } \\
\hline & & No. & $\%$ & $(95 \% \mathrm{Cl})$ & No. & $\%$ & $(95 \% \mathrm{CI})$ & No. & $\%$ & $(95 \% \mathrm{Cl})$ \\
\hline Overall & 15,976 & $|2,83|$ & 80.3 & (79.7-80.9) & 12,212 & 76.4 & (75.8-77.I) & 13,334 & 83.5 & $(82.9-84.0)$ \\
\hline \multicolumn{11}{|l|}{ Sex } \\
\hline Female & 8233 & 6611 & 80.3 & $(79.4-81.2)$ & 6257 & 76.0 & $(75.1-76.9)$ & 6802 & 82.6 & $(81.8-83.4)$ \\
\hline Male & 7743 & 6220 & 80.3 & $(79.4-81.2)$ & 5955 & 76.9 & $(76.0-77.8)$ & 6532 & 84.4 & $(83.5-85.2)$ \\
\hline \multicolumn{11}{|c|}{ Age (in years) } \\
\hline$\leq 39$ & 168 & 134 & 79.8 & $(73.2-85.3)$ & 128 & 76.2 & $(69.3-82.2)$ & $|4|$ & 83.9 & (77.8-88.9) \\
\hline $40-59$ & 2327 & 1966 & 84.5 & $(83.0-85.9)$ & 1914 & 82.3 & $(80.7-83.8)$ & 2081 & 89.4 & $(88.1-90.6)$ \\
\hline $60-79$ & 9262 & 7655 & 82.7 & $(81.9-83.4)$ & 7324 & 79.1 & (78.2-79.9) & 7950 & 85.8 & $(85.1-86.5)$ \\
\hline$\geq 80$ & 4219 & 3076 & 72.9 & $(71.6-74.2)$ & 2846 & 67.5 & $(66.0-68.9)$ & 3162 & 75.0 & $(73.6-76.2)$ \\
\hline \multicolumn{11}{|c|}{ Year of diagnosis } \\
\hline 2004 & 2555 & 2205 & 86.3 & $(84.9-87.6)$ & 2082 & 81.5 & $(80.0-83.0)$ & $2|4|$ & 83.8 & $(82.3-85.2)$ \\
\hline 2005 & 2615 & 2191 & 83.8 & (82.3-85.2) & 2074 & 79.3 & (77.7-80.8) & $2|4|$ & 81.9 & (80.4-83.3) \\
\hline 2006 & 2704 & 2223 & 82.2 & $(80.7-83.6)$ & 2132 & 78.9 & (77.3-80.4) & 2242 & 82.9 & $(81.5-84.3)$ \\
\hline 2007 & 2656 & 2104 & 79.2 & (77.6-80.7) & 2016 & 75.9 & (74.3-77.5) & 2225 & 83.8 & $(82.3-85.1)$ \\
\hline 2008 & 2706 & 2048 & 75.7 & (74.0-77.3) & 1960 & 72.4 & $(70.7-74.1)$ & 2265 & 83.7 & $(82.3-85.1)$ \\
\hline 2009 & 2740 & 2060 & 75.2 & (73.5-76.8) & 1948 & 71.1 & $(69.4-72.8)$ & 2320 & 84.7 & $(83.3-86.0)$ \\
\hline \multicolumn{11}{|c|}{ Comorbidity $^{\mathrm{a}}$} \\
\hline Low & 9617 & 7965 & 82.8 & $(82.1-83.6)$ & 7589 & 78.9 & $(78.1-79.7)$ & 8242 & 85.7 & $(85.0-86.4)$ \\
\hline Medium & 4706 & 3690 & 78.4 & $(77.2-79.6)$ & 3511 & 74.6 & (73.4-75.8) & 3822 & 81.2 & $(80.1-82.3)$ \\
\hline High & 1653 & 1176 & 71.1 & $(68.9-73.3)$ & 1112 & 67.3 & $(65.0-69.5)$ & 1270 & 76.8 & (74.8-78.8) \\
\hline
\end{tabular}

Notes: a Level of comorbidity according to the Charlson comorbidity index $(\mathrm{CCl})$ score; Low $(\mathrm{CCl}$ score $=0)$, Medium $(\mathrm{CCl}$ score $=\mathrm{I}-2)$, High $(\mathrm{CCl}$ score $\geq 3)$.

Abbreviations: $\mathrm{Cl}$, confidence interval; $\mathrm{T}$, tumour; $\mathrm{N}$, node; $\mathrm{M}$, metastasis.

\section{Rectal cancer}

Of the 8,292 rectal cancer patients diagnosed during the study period (Table 1), 40.3\% were female with a median age at diagnosis of 71 years. Median age for men was 69 years. Overall, TNM was complete for $68.1 \%$ (95\% CI:
$67.0 \%-69.1 \%$ ) of the rectal cancer cases. $M$ was the most complete stage component (84.8\% [95\% CI: 84.0\%-85.6\%]), $\mathrm{T}$ completeness was close to that of $\mathrm{M}(84.0 \%$ [95\% CI: $83.2 \%-84.8 \%]$ ), but $\mathrm{N}$ completeness was considerably lower (72.9\% [95\% CI: $72.0 \%-73.9 \%]$ ) (Table 3). Restricting to 
the $95.3 \%$ of the rectum cancer cases that were histologically verified left the overall TNM completeness nearly unchanged (69.6\% [95\% CI: 70.8\%-71.5\%]). TNM completeness was slightly higher in males compared with females and decreased with increasing age, from 78.3\% (95\% CI: 68.6\%-86.1\%) in patients $<40$ years to $49.2 \%$ (95\% CI: $46.8 \%-51.6 \%)$ in patients $\geq 80$ years. Also, TNM completeness declined with comorbidity level, from 71.4\% (95\% CI: 70.2\%-72.6\%) in patients with lowest comorbidity to $52.0 \%$ (95\% CI: $48.2 \%-55.9 \%$ ) in patients with a high comorbidity level. TNM completeness did not vary by year of diagnosis (Table 1). According to our stage algorithm, 2569 (31.0\%) rectal cancers were localized, 2350 (28.3\%) were regional, and $1633(19.7 \%)$ were distant. For a total of $1740(21.0 \%)$ rectal cancer cases, the TNM stage could not be assessed based on the available information (data not shown).

\section{Discussion}

To our knowledge, this nationwide population-based study is the first to evaluate the completeness of TNM registration of CRC in the DCR. Although the ascertaining of cancer diagnoses in the DCR is virtually complete, ${ }^{3-5}$ we found that approximately onethird of CRC patients had missing data on TNM classification. In particular, completeness declined with increasing age and level of comorbidity. Using a clinically based stage algorithm, we showed that the proportion of staged cases rose markedly.
The completeness of CRC staging in the US Surveillance, Epidemiology, and End Results, or SEER, database appears to be higher than what we observed in the DCR. Worthington et al reported that only $5.1 \%$ of colon and $7.8 \%$ of rectal cancers were unstaged during the 1991-2002 period. ${ }^{8}$ However, the SEER summary stage is computed using an algorithm that allows staging with one or two missing stage components. Although we also designed a stage algorithm that allowed some missing stage information, it might differ from the SEER template. Thus, the completenesses of TNM staging in the US and Danish registers is probably not directly comparable.

We found that TNM completeness varied substantially by age and level of comorbidity, which is in accordance with previous US studies..$^{6-8,13,14}$ In a study examining the proportion of unstaged disease at 18 cancer sites, Merill et al reported a steep increase with age. ${ }^{6}$ Likewise, marital status, race, sex, and prognosis of the cancers influenced staging. Koroukian et al reported that patients with more comprehensive needs for care (as measured by dependence of home health care and nursing home care) were two to five times as likely to be unstaged, compared with patients with fewer needs. ${ }^{13}$

TNM completeness for colon cancer in the DCR decreased slightly during the study period, whereas rectal cancer staging remained stable over time. In contrast, a number of studies have reported a decrease in the proportion of patients with unstaged CRCs over recent years. ${ }^{6-8}$ In

Table $3 \mathrm{~T}, \mathrm{~N}$, and $\mathrm{M}$ completeness for rectal cancer; overall and by sex, age, year, and comorbidity

\begin{tabular}{|c|c|c|c|c|c|c|c|c|c|c|}
\hline & \multirow{2}{*}{$\frac{\text { Total }}{\text { No. }}$} & \multicolumn{3}{|c|}{ T completeness } & \multicolumn{3}{|c|}{$\mathbf{N}$ completeness } & \multicolumn{3}{|c|}{ M completeness } \\
\hline & & No. & $\%$ & $(95 \% \mathrm{Cl})$ & No. & $\%$ & $(95 \% \mathrm{Cl})$ & No. & $\%$ & $(95 \% \mathrm{Cl})$ \\
\hline Overall & 8292 & 6964 & 84.0 & $(83.2-84.8)$ & 6048 & 72.9 & $(72.0-73.9)$ & 7032 & 84.8 & $(84.0-85.6)$ \\
\hline \multicolumn{11}{|l|}{ Sex } \\
\hline Female & 3341 & 2739 & 82.0 & $(80.7-83.3)$ & 2354 & 70.5 & $(68.9-72.0)$ & 2762 & 82.7 & $(81.4-83.9)$ \\
\hline Male & 4951 & 4225 & 85.3 & $(84.3-86.3)$ & 3694 & 74.6 & $(73.4-75.8)$ & 4270 & 86.3 & $(85.3-87.2)$ \\
\hline \multicolumn{11}{|c|}{ Age (in years) } \\
\hline$\leq 39$ & 83 & 73 & 88.0 & (79.7-93.6) & 69 & 83.1 & $(74.0-90.0)$ & 76 & 91.6 & $(84.2-96.2)$ \\
\hline $40-59$ & 1667 & 1483 & 89.0 & $(87.4-90.4)$ & 1338 & 80.3 & $(78.3-82.1)$ & 1515 & 90.9 & $(89.4-92.2)$ \\
\hline $60-79$ & 4926 & 4285 & 87.0 & $(86.0-87.9)$ & 3765 & 76.4 & $(75.2-77.6)$ & 4275 & 86.8 & $(85.8-87.7)$ \\
\hline$\geq 80$ & 1616 & 1123 & 69.5 & $(67.2-7 I .7)$ & 876 & 54.2 & $(51.8-56.6)$ & 1166 & 72.2 & $(69.9-74.3)$ \\
\hline \multicolumn{11}{|c|}{ Year of diagnosis } \\
\hline 2004 & 1339 & 1167 & 87.2 & $(85.3-88.9)$ & 998 & 74.5 & $(72.2-76.8)$ & $|12|$ & 83.7 & $(81.7-85.6)$ \\
\hline 2005 & 1284 & 1081 & 84.2 & $(82.1-86.1)$ & 926 & 72.1 & $(69.6-74.5)$ & 1086 & 84.6 & $(82.5-86.5)$ \\
\hline 2006 & 1417 & $120 \mid$ & 84.8 & $(82.8-86.6)$ & 1035 & 73.0 & $(70.7-75.3)$ & $|22|$ & 86.2 & (84.3-87.9) \\
\hline 2007 & $137 \mid$ & 1158 & 84.5 & $(82.5-86.3)$ & 1014 & 74.0 & $(71.6-76.2)$ & 1173 & 85.6 & (83.6-87.3) \\
\hline 2008 & 1442 & 1156 & 80.2 & $(78.1-82.2)$ & 1007 & 69.8 & $(67.4-72.2)$ & 1205 & 83.6 & $(81.6-85.4)$ \\
\hline 2009 & 1439 & 1201 & 83.5 & $(81.5-85.3)$ & 1068 & 74.2 & (71.9-76.4) & 1226 & 85.2 & $(83.3-87.0)$ \\
\hline \multicolumn{11}{|c|}{ Comorbidity $^{a}$} \\
\hline Low & 5464 & 4711 & 86.2 & $(85.3-87.1)$ & 4163 & 76.2 & $(75.1-77.3)$ & 4749 & 86.9 & $(86.0-87.8)$ \\
\hline Medium & 2192 & 1793 & 81.8 & $(80.1-83.4)$ & 1512 & 69.0 & $(67.0-70.9)$ & 1806 & 82.4 & $(80.8-83.9)$ \\
\hline High & 636 & 460 & 72.3 & $(68.8-75.7)$ & 373 & 58.7 & $(54.8-62.4)$ & 477 & 75.0 & (7I.5-78.3) \\
\hline
\end{tabular}

Notes: a Level of comorbidity according to the Charlson comorbidity index score $(\mathrm{CCl})$; Low $(\mathrm{CCl}$ score $=0)$, Medium $(\mathrm{CCl}$ score $=\mathrm{I}-2)$, High $(\mathrm{CCl}$ score $\geq 3)$. Abbreviations: $\mathrm{Cl}$, confidence interval; $\mathrm{T}$, tumour; $\mathrm{N}$, node; $\mathrm{M}$, metastasis. 
2004, the DCR computerized and automated the registration of incident cancer cases, facilitating fast notifications from clinicians. A potential negative consequence is that the cancer cases might be reported before the clinical workup has been finalized. However, considering the number of initiatives aiming to improve cancer control, including the implementation of Danish National Cancer Plans in 2000 and 2005, ${ }^{15,16}$ and the establishment of a comprehensive CRC database by the Danish Colorectal Cancer Group in 2001, ${ }^{19}$ one would have expected improvements in the registration of TNM stage over the study period.

A main strength of this study is its population-based design within the setting of a uniform tax-supported health care system, largely eliminating selection bias. Our study population was identified from updated nationwide registers. Although coding errors on CRC diagnoses and comorbidities cannot be ruled out, data from the DCR and the Danish National Patient Register have been found very complete and highly valid. $3,5,12$

Our study also had limitations. The completeness and accuracy of CRC diagnoses in the automated version of DCR (from 2004 on) have not been specifically validated. Moreover, we had no information on the underlying reasons for the missing information on TNM stages in the DCR, although plausible explanations include incomplete reporting and genuine difficulties on the part of the clinician or pathologist in determining the stage of the particular cancer case. For example, patients who initially received oncological therapy might not have been registered with complete details on TNM. We found that the most vulnerable patients were least likely to undergo staging, suggesting cessation of diagnostic procedures, including lymph node status, if fragility did not allow further treatment. We also observed that approximately $6 \%$ of CRC diagnoses were not histologically verified. However, although it might be expected that the majority of non-histologically verified cases pertained to patients with high comorbidity, restriction of the study population to histologically verified CRC cases yielded results quite similar to those presented.

The DCR is a valuable source for cancer research and statistics. Despite the high level of completeness of the diagnoses in this registry, we found that one-third of CRC patients had missing TNM-stage information, although the proportion of unstaged cases declined markedly with our use of a clinically based stage algorithm. However, completeness varied differentially with age and level of comorbidity, indicating that TNM data are not missing at random. This finding warrants serious consideration of the methodological implications in future epidemiological studies on cancer incidence and survival.

\section{Acknowledgment}

This study received financial support from the Regional Clinical Epidemiological Monitoring Initiative for the Central and North Denmark Regions.

\section{Disclosure}

The authors report no conflicts of interest in this work.

\section{References}

1. Ferlay J, Shin HR, Bray F, Forman D, Mathers C, Parkin DM. Estimates of worldwide burden of cancer in 2008: GLOBOCAN 2008. Int J Cancer. 2010;127(12):2893-2917.

2. Greene FL, Page DL, Fleming ID, et al. AJCC cancer staging manual, 6th edition. New York: Springer; 2002.

3. Storm HH, Michelsen EV, Clemmensen IH, Pihl J. The Danish Cancer Registry-history, content, quality and use. Dan Med Bull. 1997;44(5):535-539.

4. Gjerstorff ML. The Danish Cancer Registry. Scand J Public Health. 2011;39(7 Suppl):42-45.

5. Jensen AR, Overgaard J, Storm HH. Validity of breast cancer in the Danish Cancer Registry. A study based on clinical records from one county in Denmark. Eur J Cancer Prev. 2002;11(4):359-364.

6. Merrill RM, Sloan A, Anderson AE, Ryker K. Unstaged cancer in the United States: a population-based study. BMC Cancer. 2011;11:402.

7. Klassen AC, Curriero F, Kulldorff M, Alberg AJ, Platz EA, Neloms ST. Missing stage and grade in Maryland prostate cancer surveillance data, 1992-1997. Am J Prev Med. 2006;30(2 Suppl):S77-87.

8. Worthington JL, Koroukian SM, Cooper GS. Examining the characteristics of unstaged colon and rectal cancer cases. Cancer Detect Prev. 2008;32(3):251-258.

9. Pedersen CB. The Danish Civil Registration System. Scand J Public Health. 2011;39(7 Supp1):22-25.

10. Lynge E, Sandegaard JL, Rebolj M. The Danish National Patient Register. Scand J Public Health. 2011;39(7 Suppl):30-33.

11. Charlson ME, Pompei P, Ales KL, MacKenzie CR. A new method of classifying prognostic comorbidity in longitudinal studies: development and validation. J Chronic Dis. 1987;40(5):373-383.

12. Thygesen SK, Christiansen CF, Christensen S, Lash TL, Sorensen HT. The predictive value of ICD-10 diagnostic coding used to assess Charlson comorbidity index conditions in the population-based Danish National Registry of Patients. BMC Med Res Methodol. 2011;11:83.

13. Koroukian SM, Xu F, Beaird H, Diaz M, Murray P, Rose JH. Complexity of care needs and unstaged cancer in elders: a population-based study. Cancer Detect Prev. 2007;31(3):199-206.

14. Yancik R, Wesley MN, Ries LA, Havlik RJ, Edwards BK, Yates JW. Effect of age and comorbidity in postmenopausal breast cancer patients aged 55 years and older. JAMA. 2001;285(7):885-892.

15. National Board of Health. Evaluation of the Danish National Cancer Action Plan: status and future monitoring — summary and proposals for focus areas. Copenhagen: The National Board of Health; 2004.

16. National Board of Health. National cancer plan II-Denmark: National Board of Health recommendations for improving cancer healthcare services. Version: 1.0 ed. Copenhagen: The National Board of Health; 2005.

17. Danish Colorecal Cancer Group. Landsdækkende database for kræft i tyktarm og endetarm: Årsrapport 2010 [Nationwide database of colorectal cancer: annual report 2010]. Copenhagen: DCCG; 2011. Danish. 


\section{Appendix I}

Appendix I Algorithm for colorectal cancer (CRC) staging according to the TNM classification

\begin{tabular}{|c|c|}
\hline Tumor stage & TNM $^{a}$ \\
\hline \multirow[t]{3}{*}{ Localized } & TI-4,x NO MO \\
\hline & TI-2 N0 Mx \\
\hline & TI Nx M0,x \\
\hline Regional & TI-4,x NI-2 MO \\
\hline Distant & $\mathrm{TI}-4, \mathrm{x}$ N0-2,x MI \\
\hline \multirow[t]{3}{*}{ Unknown ${ }^{\mathrm{b}}$} & $\mathrm{T} 2-4, \mathrm{x} N \times \mathrm{M} 0, \mathrm{x}$ \\
\hline & T3-4,x No Mx \\
\hline & TI-4,x NI-2 Mx \\
\hline
\end{tabular}

Notes: aln all, 466 CRC cases were assigned N3 (categorized as N2 in the algorithm); ${ }^{\mathrm{b}}$ Thirty CRC cases were assigned T0, Ta, or Tis (categorized as unknown stage in the algorithm).

Abbreviation: TNM, tumor, node, metastasis.

\section{Publish your work in this journal}

Clinical Epidemiology is an international, peer-reviewed, open access journal focusing on disease and drug epidemiology, identification of risk factors and screening procedures to develop optimal preventative initiatives and programs. Specific topics include: diagnosis, prognosis, treatment, screening, prevention, risk factor modification, systematic

Submit your manuscript here: http://www.dovepress.com/clinical-epidemiology-journal reviews, risk \& safety of medical interventions, epidemiology \& biostatical methods, evaluation of guidelines, translational medicine, health policies \& economic evaluations. The manuscript management system is completely online and includes a very quick and fair peer-review system, which is all easy to use. 